\title{
AN ANALOG OF THOMPSON'S TRIANGLE INEQUALITY IN EUCLIDEAN JORDAN ALGEBRAS*
}

\author{
J. $\mathrm{TAO}^{\dagger}$
}

\begin{abstract}
In a recent paper [Linear Algebra Appl., 461:92-122, 2014], Tao et al. proved an analog of Thompson's triangle inequality for a simple Euclidean Jordan algebra by using a case-by-case analysis. In this short note, we provide a direct proof that is valid on any Euclidean Jordan algebras.
\end{abstract}

Key words. Euclidean Jordan algebra, Majorization, Thompson's triangle inequality.

AMS subject classifications. 15A42, 17C20, 17C55.

1. Introduction. In matrix theory, the well-known Thompson's triangle asserts that for any two complex square matrices $A$ and $B$ of the same size, there exist unitary matrices $U$ and $V$ such that

$$
|A+B| \leq U|A| U^{*}+V|B| V^{*},
$$

where $|A|$ is the (unique) square root of $A A^{*}$.

In a recent paper, Tao et al. [4] extended this by proving the following result: in a simple Euclidean Jordan algebra $V$, for any two elements $a, b \in V$, there exist $\Lambda, \Lambda^{\prime} \in \operatorname{Aut}(V)$ such that

$$
|a+b| \leq \Lambda(|a|)+\Lambda^{\prime}(|b|),
$$

where $\operatorname{Aut}(V)$ is the set of algebra automorphisms on $V$.

The result was proved by considering each of the five simple algebras. The main objective of this note is to offer a direct proof for any Euclidean Jordan algebra. The novelty here is the direct proof based on general results on Euclidean Jordan algebras.

2. Preliminaries. Throughout this paper, we let $(V, \circ,\langle\cdot, \cdot\rangle)$ denote an Euclidean Jordan algebra of rank $r$ with unit element $e$ and $K:=\{x \circ x: x \in V\}$ be the cone of squares in $V$ [1], the Jordan product and inner product of elements $x$ and $y$ in $V$ are, respectively, denoted by $x \circ y$ and $\langle x, y\rangle$. It is well known [1] that any Euclidean Jordan algebra is a direct product/sum of simple Euclidean Jordan algebras and every simple Euclidean Jordan algebra is isomorphic to one of five algebras, three of which are the algebras of $n \times n$ real/complex/quaternion Hermitian matrices. The other two are the algebra of $3 \times 3$ octonion Hermitian matrices and the Jordan spin algebra. In the algebras $\mathcal{S}^{n}$ (of all $n \times n$ real symmetric matrices) and $\mathcal{H}^{n}$ (of all $n \times n$ complex Hermitian matrices), the Jordan product and the inner product are given, respectively, by

$$
X \circ Y:=\frac{X Y+Y X}{2} \text { and }\langle X, Y\rangle:=\operatorname{tr}(\mathrm{XY})
$$

where the trace of a real/complex matrix is the sum of its diagonal entries.

\footnotetext{
*Received by the editors on August 4, 2020. Accepted for publication on January 26, 2021. Handling Editor: Tin-Yau Tam.

$\dagger$ Department of Mathematics and Statistics, Loyola University Maryland, Baltimore, Maryland 21210, USA (jtao@ loyola.edu).
} 
Electronic Journal of Linear Algebra, ISSN 1081-3810

A publication of the International Linear Algebra Society

Volume 37, pp. 156-159, January 2021.

In addition, the (trace) inner product is defined by $\langle x, y\rangle:=\operatorname{tr}(x \circ y)$ for any $x, y \in V$. In $V$, one can define the linear automorphism group $\operatorname{Aut}(V)$ in the following way (see [1]): $\Lambda \in A u t(V)$ if $\Lambda: V \rightarrow V$ is invertible and $\Lambda(x \circ y)=\Lambda(x) \circ \Lambda(y)$ for all $x, y \in V$. We use the notation $x \geq 0(x>0)$ when $x \in K$ (respectively, $x \in K^{o}$ (=interior $\left.(K)\right)$ ). An element $c \in V$ is an idempotent if $c^{2}=c$; it is said to be a primitive idempotent if it is nonzero and cannot be written as the sum of two other nonzero idempotents.

Given an idempotent $c$, we have the Peirce (orthogonal) decomposition [1] $V=V(c, 1)+V\left(c, \frac{1}{2}\right)+V(c, 0)$, where $V(c, \gamma)=\{x \in V: x \circ c=\gamma x\}$ with $\gamma \in\left\{1, \frac{1}{2}, 0\right\}$. Then, any $b \in V$ can be decomposed as $b=u+v+w$, where $u \in V(c, 1), v \in V\left(c, \frac{1}{2}\right)$ and $w \in V(c, 0)$. We say that $u$ is the principal component of $b$ relative to $c$.

The spectral decomposition. For $x \in V$, there exists a Jordan frame $\left\{e_{1}, \ldots, e_{r}\right\}$ and real numbers $\lambda_{1}(x), \ldots, \lambda_{r}(x)$ such that

$$
x=\lambda_{1}(x) e_{1}+\cdots+\lambda_{r}(x) e_{r} .
$$

The numbers $\lambda_{i}(x)$ are called the eigenvalues of $x$.

Given (2.3), $|x|=\sum_{i=1}^{r}\left|\lambda_{i}(x)\right| e_{i}$ and the trace of $x$ is $\operatorname{tr}(x)=\langle x, e\rangle=\sum_{i=1}^{r} \lambda_{i}(x)$ for all $x \in V$.

Throughout, given a vector $x=\left(x_{1}, x_{2}, \ldots, x_{r}\right)$ in $\mathbb{R}^{r}$, we write $x^{\downarrow}:=\left(x_{1}^{\downarrow}, x_{2}^{\downarrow}, \ldots, x_{r}^{\downarrow}\right)$ for the vector obtained by rearranging the components of $x$ in the decreasing order. For two vectors $x=\left(x_{1}, x_{2}, \ldots, x_{r}\right)$ and $y=\left(y_{1}, y_{2}, \ldots, y_{r}\right)$ in $\mathbb{R}^{r}$, we say that $x$ is weekly majorized by $y$ and write $x \prec_{w} y$ if for each index $k$, $1 \leq k \leq n, \sum_{1}^{k} x_{i}^{\downarrow} \leq \sum_{1}^{k} y_{i}^{\downarrow}$; additionally, if the equality holds when $k=r$, we say that $x$ is majorized by $y$ and write $x \prec y$. For $x \in V$, let $\lambda(x)$ denote the vector of eigenvalues of $x$ written in the decreasing order.

Now we recall some known results used in this paper.

Let $\left\{e_{1}, e_{2}, \ldots, e_{r}\right\}$ be a Jordan frame. For $1 \leq k \leq r$, let

$$
V^{(k)}=V_{\left\{e_{1}, e_{2}, \ldots, e_{k}\right\}}:=\left\{x \in V: x \circ\left(e_{1}+e_{2} \cdots+e_{k}\right)=x\right\} .
$$

For any $z \in V$, we let

$$
\bar{z}:=P_{e_{1}+e_{2}+\cdots+e_{k}}(z)=\sum_{1}^{k} z_{i} e_{i}+\sum_{1 \leq i<j \leq k} z_{i j} .
$$

We call $\bar{z}$, the principal component of $z$ corresponding to $\left\{e_{1}, e_{2}, \ldots, e_{k}\right\}$.

TheOREm 2.1 (see Theorem 4.2, [3]). Let $V$ be simple and $\left\{e_{1}, e_{2}, \ldots, e_{r}\right\}$ be a Jordan frame. For any element $z \in V$, let $\bar{z}$ denote the principal component of $z$ with respect to $\left\{e_{1}, e_{2}, \ldots, e_{k}\right\}$. Then,

$$
\lambda_{i}(z) \geq \lambda_{i}(\bar{z}) \geq \lambda_{r-k+i}(z) \quad(i=1,2, \ldots, k)
$$

Lemma 2.2 (Lemma 3.1, [4]). Let $V$ be simple. Suppose that $\left(\lambda_{1}(x), \lambda_{2}(x), \ldots, \lambda_{r}(x)\right)$ and $\left(\lambda_{1}(y)\right.$, $\left.\lambda_{2}(y), \ldots, \lambda_{r}(y)\right)$ are eigenvalues (not necessarily in the decreasing order) for $x \in V$ and $y \in V$, respectively. If $\lambda_{i}(x) \leq \lambda_{i}(y), i=1,2, \ldots, r$, then there exists a $\Lambda \in \operatorname{Aut}(V)$ such that $x \leq \Lambda(y)$.

Proposition 2.3. Let $x, y \in V$. Then,

(i) $\lambda(x+y) \prec \lambda(x)+\lambda(y)$.

(ii) $x \leq y \Rightarrow \lambda(x) \leq \lambda(y)$.

In the above proposition, Item (i) is stated in [2], Proposition 8. Item (ii) is a consequence of the well-known min-max theorem of Hirzebruch [3]. 
3. Results. In $V$, consider an element $\varepsilon$ such that $\varepsilon^{2}=e$. Then, we could express $\varepsilon=\sum_{i=1}^{r} \varepsilon_{i} e_{i}$, where $\varepsilon_{i}=1$ for $i=1,2, \ldots, k$ and $\varepsilon_{j}=-1$ for $j=k+1, k+2, \ldots, r(1 \leq k \leq r)$ for a fixed Jordan frame $\left\{e_{1}, \ldots, e_{r}\right\}$.

Lemma 3.1. Let $V$ be simple with rank $r$. Then, for $x \in V$ and $\varepsilon$ with $\varepsilon^{2}=e$, there exists a $\Lambda \in$ Aut $(V)$ such that $x \circ \varepsilon \leq \Lambda(|x|)$.

Proof. As the result is obvious when $\varepsilon=e$ or $-e$, we assume the spectral decomposition of $\varepsilon$ in the form $\varepsilon=\sum_{i=1}^{r} \varepsilon_{i} e_{i}$, where $1 \leq k<r, \varepsilon_{i}=1$ for $i=1,2, \ldots, k$ and $\varepsilon_{j}=-1$ for $j=k+1, k+2, \ldots, r$. We let $c=\sum_{1}^{k} e_{i}$ and consider the Peirce decomposition $x=u+v+w$, where $u \in V(c, 1), v \in V\left(c, \frac{1}{2}\right)$ and $w \in V(c, 0)$. A direct calculation leads to $x \circ \varepsilon=u-w$. Now, writing the spectral decompositions of $u$ and $w$ in the (sub)algebras $V(c, 1)$ and $V(c, 0)$ in the form $u=\sum_{1}^{k} u_{i} f_{i}$ and $w=\sum_{k+1}^{n} w_{i} f_{i}$ for some Jordan frame $\left\{f_{1}, f_{2}, \ldots, f_{n}\right\}$ in $V$, we see that eigenvalues of $x \circ \varepsilon$ are $\lambda_{i}(u)(1 \leq i \leq k)$ and $\lambda_{j}(-w)(1 \leq j \leq r-k)$. We note that $u(-w)$ is principal component of $x(-x)$. By Theorem 2.1, $\lambda_{i}(u) \leq \lambda_{i}(x) \leq\left|\lambda_{i}(x)\right|$ for $1 \leq i \leq k$ and $\lambda_{i}(-w) \leq \lambda_{i}(-x)=-\lambda_{r-i+1}(x) \leq\left|\lambda_{r-i+1}(x)\right|$ for $1 \leq i \leq r-k$, which implies $\lambda(x \circ \varepsilon) \leq \sigma_{1}|\lambda(x)|=$ $\sigma_{1} \sigma(\lambda(|x|))$, where $\sigma$ and $\sigma_{1}$ are permutation matrices. By Lemma 2.2, there exists a $\Lambda \in A u t(V)$ such that $x \circ \varepsilon \leq \Lambda(|x|)$.

TheOREm 3.2. Let $V$ be any Euclidean Jordan algebra. Then, for $a, b \in V$, there exist $\Lambda, \Lambda^{\prime} \in$ Aut $(V)$ such that

$$
|a+b| \leq \Lambda(|a|)+\Lambda^{\prime}(|b|) .
$$

Proof. First, suppose that $V$ is simple. Let $a+b=\sum_{i=1}^{k} \lambda_{i}(a+b) e_{i}$. Without loss of generality, we assume that $\lambda_{i}(a+b) \geq 0$, for $i=1,2, \ldots, k$ and $\lambda_{j}(a+b)<0$ for $j=k+1, k+2, \ldots, r$. Now, let $\varepsilon=\sum_{i=1}^{r} \varepsilon_{i} e_{i}$, where $\varepsilon_{i}=1$ for $i=1,2, \ldots, k$ and $\varepsilon_{j}=-1$ for $j=k+1, k+2, \ldots, r$. Then,

$$
|a+b|=(a+b) \circ \varepsilon=a \circ \varepsilon+b \circ \varepsilon .
$$

By Lemma 3.1, there exist $\Lambda, \Lambda^{\prime} \in A u t(V)$ such that $a \circ \varepsilon \leq \Lambda(|a|)$ and $b \circ \varepsilon \leq \Lambda^{\prime}(|b|)$. Thus,

$$
|a+b| \leq \Lambda(|a|)+\Lambda^{\prime}(|b|) .
$$

Now suppose that $V$ is not simple, without loss of generality, we let $V=V_{1} \times V_{2}$, where each $V_{i}$ is a simple algebra. For $a=\left(a_{1}, a_{2}\right) \in V, a_{i} \in V_{i}$, and $b=\left(b_{1}, b_{2}\right) \in V, b_{i} \in V_{i}$,

$$
|a+b|=\left[\begin{array}{c}
\left|a_{1}+b_{1}\right| \\
\left|a_{2}+b_{2}\right|
\end{array}\right] \leq\left[\begin{array}{c}
\Lambda_{1}\left(\left|a_{1}\right|\right)+\Lambda_{1}^{\prime}\left(\left|b_{1}\right|\right) \\
\Gamma\left(\left|a_{2}\right|\right)+\Gamma^{\prime}\left(\left|b_{2}\right|\right)
\end{array}\right]=\left[\begin{array}{cc}
\Lambda & 0 \\
0 & \Gamma
\end{array}\right]\left[\begin{array}{c}
\left|a_{1}\right| \\
\left|a_{2}\right|
\end{array}\right]+\left[\begin{array}{cc}
\Lambda_{1}^{\prime} & 0 \\
0 & \Gamma^{\prime}
\end{array}\right]\left[\begin{array}{l}
\left|b_{1}\right| \\
\left|b_{2}\right|
\end{array}\right]
$$

where $\Lambda_{1}, \Lambda_{1}^{\prime} \in \operatorname{Aut}\left(V_{1}\right)$, and $\Gamma, \Gamma^{\prime} \in \operatorname{Aut}\left(V_{2}\right)$. It is clear that $\Lambda:=\left[\begin{array}{cc}\Lambda & 0 \\ 0 & \Gamma\end{array}\right] \in \operatorname{Aut}(V)$ and $\Lambda^{\prime}:=$ $\left[\begin{array}{cc}\Lambda_{1}^{\prime} & 0 \\ 0 & \Gamma^{\prime}\end{array}\right] \in \operatorname{Aut}(V)$. Therefore, $|a+b| \leq \Lambda(|a|)+\Lambda^{\prime}(|b|)$.

REMARK 3.3. In view of the previous longer and more complicated proof of Theorem 3.1 in [4], the proof of Theorem 3.2 is much shorter and more general (for any Euclidean Jordan algebra). This is due to Lemma 3.1. The key idea there is to introduce such an $\varepsilon$ such that $x \circ \varepsilon$ happens to be principal components of $x$ and $-x$ in the (sub)algebras $V(c, 1)$ and $V(c, 0)$. Then, thanks Cauchy interlacing theorem (Theorem $2.1)$, the corresponding eigenvalue inequalities can be established.

The above theorem immediately yields the following corollary. 
Corollary 3.4. Let $V$ be any Euclidean Jordan algebra and $a, b \in V$. Then,

$$
\lambda(|a+b|) \underset{w}{\prec} \lambda(|a|)+\lambda(|b|) .
$$

Proof. By Theorem 3.2, for any $a, b \in V$, there exist $\Lambda, \Lambda^{\prime} \in A u t(V)$ such that $|a+b| \leq \Lambda(|a|)+\Lambda^{\prime}(|b|)$. By Item (ii) in Proposition 2.3, $\lambda_{i}(|a+b|) \leq \lambda_{i}\left(\Lambda(|a|)+\Lambda^{\prime}(|b|)\right)$ for all $i$. Now, by Item (i) in Proposition 2.3 , we have

$$
\lambda\left(\Lambda(|a|)+\Lambda^{\prime}(|b|)\right) \prec \lambda(\Lambda(|a|))+\lambda\left(\Lambda^{\prime}(|b|)\right)=\lambda(|a|)+\lambda(|b|) .
$$

Thus, $\lambda(|a+b|) \underset{w}{\prec} \lambda(|a|)+\lambda(|b|)$.

4. Applications. A function $\phi: \mathbb{R} \rightarrow \mathbb{R}$ is said to be subadditive if $\phi(t+s) \leq \phi(t)+\phi(s)$ for all $t, s \in \mathbb{R}$. An example is $\phi(t)=\sqrt{t}$.

THEOREM 4.1. Let $V$ be any Euclidean Jordan algebra and $a, b \in V$. If $f$ is nonnegative increasing subadditive function on $[0, \infty)$, then for all $i$,

$$
f\left(\lambda_{i}(|a+b|)\right) \leq f\left(\lambda_{i}(|a|)\right)+f\left(\lambda_{i}(|b|)\right)
$$

Proof. By Theorem 3.2, for any $a, b \in V$, there exist $\Lambda, \Lambda^{\prime} \in A u t(V)$ such that $|a+b| \leq \Lambda(|a|)+\Lambda^{\prime}(|b|)$. This implies that $\lambda_{i}(|a+b|) \leq \lambda_{i}\left(\Lambda(|a|)+\Lambda^{\prime}(|b|)\right)$ for all $i$ (see Item (ii) in Proposition 2.3). Since $f$ is increasing function, we have for all $i$,

$$
f\left(\lambda_{i}(|a+b|)\right) \leq f\left(\lambda_{i}\left(\Lambda(|a|)+\Lambda^{\prime}(|b|)\right)\right) \leq f\left(\lambda_{i}(\Lambda(|a|))+f\left(\lambda_{i}\left(\Lambda^{\prime}(|b|)\right)=f\left(\lambda_{i}(|a|)\right)+f\left(\lambda_{i}(|b|)\right) .\right.\right.
$$

Note that the second inequality is due to the subadditivity of $f$.

Corollary 4.2. Let $V$ be an any Euclidean Jordan algebra and $a, b \in V$. Then, for all $i$,

$$
\sqrt{\left.\lambda_{i}(|a+b|)\right)} \leq \sqrt{\left.\lambda_{i}(|a|)\right)}+\sqrt{\left.\lambda_{i}(|b|)\right)}
$$

Proof. Since $f(t)=\sqrt{t}$ is nonnegative increasing subadditive function on $[0, \infty)$, by Theorem 4.2 , (4.5) holds.

Acknowledgment. We would like to thank the anonymous referee for his/her very constructive suggestions and comments.

\section{REFERENCES}

[1] J. Faraut and A. Koranyi. Analysis on Symmetric Cones. Oxford University Press, Oxford, 1994.

[2] J. Jeong and M.S. Gowda. Spectral sets and functions in Euclidean Jordan algebras. Linear Algebra Appl., 518:31-56, 2017.

[3] M.S. Gowda and J. Tao. The Cauchy interlacing theorem in simple Euclidean Jordan algebras and some consequences. Linear Multilinear Algebra, 59:65-86, 2011.

[4] J. Tao, L. Kong, Z. Luo, and N. Xiu. Some majorization inequalities in Euclidean Jordan algebras. Linear Algebra Appl., 461:92-122, 2014. 